\title{
Nutrición
}

\section{Diagnóstico del estado nutricional y su impacto en el tratamiento del cáncer}

L. Cerezo

En los últimos años estamos asistiendo en oncología a un creciente interés por los tratamientos de soporte en general y por el soporte nutricional en particular. La razón fundamental está en la relación que existe entre malnutrición y un peor pronóstico de los pacientes tumorales, conocida desde hace varios años.

La enfermedad y la nutrición están íntimamente relacionadas, de modo que, mientras la enfermedad puede causar desnutrición, la desnutrición puede, a su vez, influir negativamente en la enfermedad'. La mayoría de los oncólogos sabemos que es crucial mantener un buen estado nutricional durante el proceso terapéutico de los pacientes con cáncer pero todavía somos poco conscientes de las estrategias nutricionales a nuestro alcance.

Los objetivos del soporte nutricional en oncología son mejorar la tolerancia al tratamiento oncológico específico, disminuir la incidencia de complicaciones, aumentar el control tumoral y ante todo mejorar la calidad de vida del paciente. El diagnóstico del estado nutricional deberá integrarse en la valoración global del paciente y ser una prioridad en su plan de terapéutico. Es necesario un abordaje multidisciplinario que implique al oncólogo, al especialista en nutrición, al paciente y a su familia o allegados. El oncólogo clínico debe sensibilizarse sobre la importancia de evaluar el estado nutricional del paciente y actuar sobre las alteraciones del metabolismo en fases precoces.

Toda intervención nutricional debe empezar por una valoración nutricional apropiada del paciente, realizada a poder ser en el momento del diagnóstico de cáncer y a intervalos regulares durante su enfermedad. Si esto no es posible, la valoración nutricional se hará a la llegada del paciente al servicio de oncología radioterápica y durante todo el tratamiento radioterápico o radioquimioterápico.

Para poder identificar a los pacientes malnutridos necesi-

Servicio de Oncología Radioterápica Hospital Universitario de la Princesa Madrid tamos herramientas que traduzcan fielmente la situación nutricional de la persona evaluada, que no sean costosas, y que puedan realizarse de forma rápida, sencilla y reproducible. En la práctica clínica, el objetivo fundamental de la valoración nutricional es identificar a pacientes con malnutrición o con riesgo aumentado de complicaciones nutricionales por su propia enfermedad o los tratamientos aplicados, tales como la cirugía, la radioterapia o la quimioterapia, que se beneficiarían de un adecuado abordaje nutricional. Por tanto, la valoración nutricional inicial ayuda a discriminar qué pacientes requieren actuación nutricional y permite una adecuada monitorización del estado nutricional durante el tratamiento e incluso una vez finalizado el mismo, durante el seguimiento evolutivo, si el paciente presenta secuelas relacionadas con la nutrición.

Básicamente disponemos de dos tipos de herramientas para realizar el diagnóstico o valoración nutricional, los métodos tradicionales y los llamados métodos rápidos de valoración del estado nutricional, que pasaremos a exponer a continuación.

\section{Métodos tradicionales de valoración del estado nutricional}

Los métodos tradicionales de valoración del estado nutricional de una persona se basan en la medición de parámetros antropométricos, concentración plasmática de proteínas, composición corporal y determinación de índices pronósticos. Hay que tener en cuenta que estas medidas pueden verse alteradas en algunas circunstancias ajenas a los problemas nutricionales, como son la edad o el estado de hidratación entre otros.

\section{Antropometría}

El peso y la talla son las medidas antropométricas más sencillas de obtener y suponen un medio preciso, rápido y reproducible de valoración nutricional. Se deben tener en cuenta la ropa y el calzado, así como la presencia de edemas, ascitis o una gran masa tumoral que limitarían la utilidad del peso como parámetro de valoración nutricional, ya que pueden enmascarar una depleción de masa corporal. Mediante tablas específicas se puede obtener el peso ideal a través de la talla, sexo y la edad del paciente. 
El índice de masa corporal (IMC) es una medida que relaciona el peso con la talla, mediante la siguiente ecuación: $I M C=$ peso $(\mathrm{kg}) /$ talla $^{2}\left(\mathrm{~m}^{2}\right)$. Como el peso se afecta más que la talla por el estado nutricional, se aumenta el valor relativo de la talla elevándola al cuadrado. Se consideran valores normales un IMC comprendido entre 20 y $25 \mathrm{~kg} / \mathrm{m}^{2}$, bajo peso entre 20 y $18,5 \mathrm{~kg} / \mathrm{m}^{2}$ y malnutrición por debajo de $18,5 \mathrm{~kg} / \mathrm{m}^{2}$.

De suma importancia en oncología es hacer referencia al cambio de peso que se ha producido con respecto al habitual y su evolución en el tiempo. La pérdida de peso se valora del siguiente modo:

pérdida de peso $(\%)=\frac{\text { (peso habitual }- \text { peso actual }) \times 100}{\text { peso habitual }}$

Una pérdida de peso superior a $10 \%$ de forma involuntaria en un período de tiempo inferior a 6 meses es un criterio de primera línea de malnutrición, teniendo significado pronóstico en los pacientes con cáncer ${ }^{2,3}$. El riesgo de complicaciones aumenta con la cuantía de la pérdida y la velocidad de instauración de la misma. Sin embargo, en qué grado la pérdida de masa corporal por sí misma contribuye directamente a la mala evolución de la enfermedad o simplemente refleja la gran alteración del metabolismo inducido en el huésped por el tumor, es difícil de deducir ${ }^{4}$.

La medida de los pliegues cutáneos y la circunferencia muscular del brazo son también un métodos aceptables para valorar la grasa corporal y el compartimento muscular. El pliegue cutáneo tricipital es el más empleado y estima la masa grasa periférica. Se mide colocando el lipocalibrador en el punto medio de la cara posterior del brazo en extensión y relajado, entre el acromion y el olécranon, con el pliegue paralelo al eje longitudinal del brazo. Es conveniente realizar el proceso tres veces y promediar los valores obtenidos en milímetros. Sobretodo son útiles para hacer una valoración evolutiva, si disponemos de medidas previas para compararlos. Sin embargo, no son útiles para valorar cambios a corto plazo, y sus valores pueden verse artefactados por la presencia de edemas.

\section{Impedanciometría}

La impedanciometría se basa en la aplicación de una corriente eléctrica de bajos potenciales e intensidad a distintas frecuencias que se transmite de forma distinta a través de los tejidos magros y adiposos, siendo mayor la conducción por el primero. Se detectan en los electrodos impedancia, resistencia y reactancia y, a partir de éstas, las masas grasas, magra y el contenido de agua. Se considera una masa adiposa insuficiente cuando el porcentaje de grasa corporal es inferior al $12 \%$ en hombres y $20 \%$ en mujeres 5 .

Existen otros métodos investigacionales para estimar los distintos compartimentos del organismo basados en el rastreo isotópico, densitometría o la determinación del potasio corporal total. La dinamometría consiste en la medición de la fuerza de aprehensión de la mano por un dinamómetro. Es un método de valoración funcional del componente muscular esquelético. Su relación con el estado nutricional del paciente no está clara pero se ha demostrado su valor como índice pronóstico en pacientes sometidos a cirugía ${ }^{6}$.

\section{Concentración plasmática de proteínas}

Se emplean para valorar el compartimento proteico visceral y habitualmente se determinan albúmina, transferrina, prealbúmina y/o proteína transportadora del retinol. Todas son sintetizadas en el hígado y no dependen únicamente del estado nutricional, sino que se modifican también en otras situaciones que hay que descartar, como cirugía, traumatismos, infecciones y otros procesos agudos.

La albúmina es un proteína encargada de transportar en el plasma múltiples sustancias y mantener la presión oncótica de la sangre. Su vida media es larga (aproximadamente 18 días), por lo que es poco sensible a modificaciones recientes del estado nutricional, de modo que puede mantenerse normal durante bastante tiempo a pesar de un déficit nutricional importante. De igual forma, es posible encontrar un descenso plasmático de albúmina sin que exista un défict nutricional asociado en caso de enfermedad hepática, síndrome nefrótico o enteropatía pierde proteínas. En situaciones de expansión de volumen puede observarse una hipoalbuninemia por dilución. A pesar de todo es muy útil para valorar situaciones de desnutrición crónicas. La albuminemia es el mejor índice de laboratorio en la evaluación nutricional inicial de los pacientes, ya que tiene un alto valor predictivo de complicaciones asociadas a la desnutrición. Cifras inferiores a $25 \mathrm{~g} / \mathrm{l}$ sugieren un elevado riesgo de complicaciones.

La prealbúmina es más sensible que la anterior para detectar cambios en el estado nutricional porque tiene una vida media más corta (2 días). Se eleva rápidamente en respuesta al tratamiento nutricional, y tiene una buena correlación con el balance nitrogenado. Un descenso en los niveles de preal-

TABLA I

Vida media y valores plasmáticos de las proteínas viscerales

\begin{tabular}{lccccc}
\hline Proteína & $\begin{array}{c}\text { Vida } \\
\text { Media }\end{array}$ & $\begin{array}{c}\text { Valor } \\
\text { normal }\end{array}$ & $\begin{array}{c}\text { Depleción } \\
\text { leve }\end{array}$ & $\begin{array}{c}\text { Depleción } \\
\text { moderada }\end{array}$ & $\begin{array}{c}\text { Depleción } \\
\text { grave }\end{array}$ \\
\hline Albúmina $(\mathrm{g} / \mathrm{dl})$ & $18-20 \mathrm{~d}$ & $3,5-4,5$ & $2,8-3,4$ & $2,1-2,7$ & $<2,1$ \\
Transferrina $(\mathrm{mg} / \mathrm{dl})$ & $8-10 \mathrm{~d}$ & $250-350$ & $150-250$ & $100-150$ & $<-100$ \\
Prealbúmina $(\mathrm{mg} / \mathrm{dl})$ & $2 \mathrm{~d}$ & $18-28$ & $15-18$ & $10-15$ & $<10$ \\
RBP $(\mathrm{mg} / \mathrm{dl})$ & $12 \mathrm{~h}$ & $2,6-7$ & $2-2,6$ & $1,5-2$ & $<1,5$ \\
\hline
\end{tabular}

RBP: proteína transportadora del retinol 
búmina se acompaña de complicaciones hasta en un $40 \%$ de los casos. Sin embargo, se trata también de un reactante negativo de fase aguda $y$, por tanto, disminuye en caso de infección, traumatismo, cirugía, etc. En situaciones de insuficiencia renal puede verse aumentada su concentración plasmática, ya que tiene una excreción principalmente renal. Su respuesta al tratamiento nutricional es un excelente factor pronóstico de supervivencia en el cáncer ${ }^{7}$.

La transferrina tiene una vida media menor que la albú- mina (8 días), pudiendo valorar cambios en el estado nutricional superiores a una semana. Su función es transportar el hierro, por lo que alteraciones en su metabolismo afectan a los niveles de la proteína y disminuyen los valores de ésta en la insuficiencia renal y las infecciones crónicas. Es más útil en el seguimiento de los pacientes que en la valoración nutricional inicial, ya que las modificaciones en su concentración se correlacionan positivamente con el balance nitrogenado.

Por favor, conteste al siguiente formulario escribiendo los datos que se le piden o señalando la opción correcta, cuando se le ofrecen varias

\begin{tabular}{|l|}
\hline Nombres y Apellidos \\
\hline PESO actual \\
Peso hace 3 meses $\_\mathrm{kg}$ \\
$\mathrm{kg}$
\end{tabular}

ALIMENTACIÓN respecto hace 1 mes:

$$
\begin{aligned}
& \square \text { como más } \\
& \square \text { como igual } \\
& \square \text { como menos }
\end{aligned}
$$

Tipo de alimentos:

$$
\begin{aligned}
& \square \text { dieta normal } \\
& \square \text { pocos sólidos } \\
& \square \text { sólo líquidos } \\
& \square \text { sólo preparados nutricionales } \\
& \square \text { muy poco }
\end{aligned}
$$

ALIMENTACIÓN respecto hace 1 mes:

$$
\begin{aligned}
& \square \text { normal } \\
& \text { menor de lo habitual } \\
& \text { sin ganas de nada } \\
& \text { paso mas de la mitad del día en cama o } \\
& \text { sentado }
\end{aligned}
$$

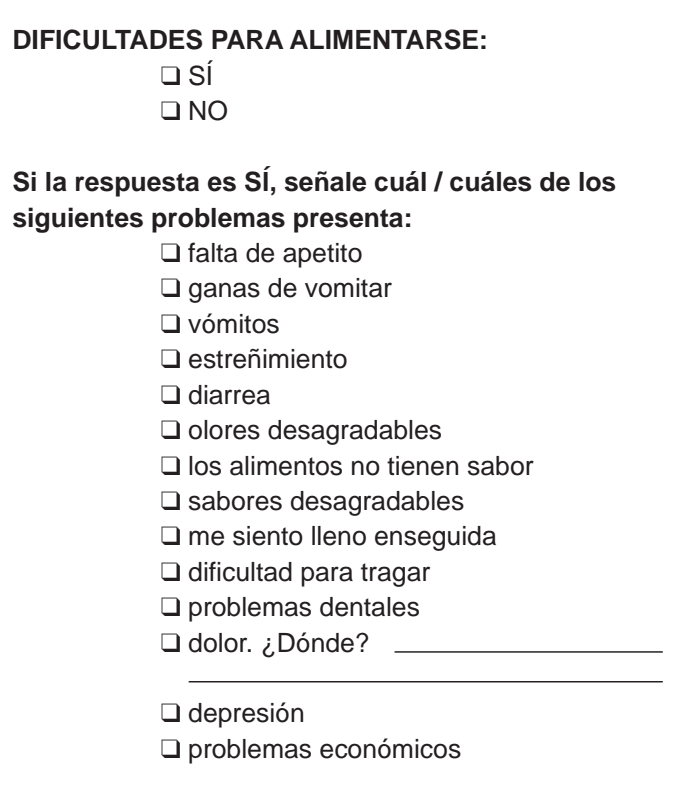

Muchas Gracias. A partir de aquí, lo completará su médico.

\section{ENFERMEDADES:}

TRATAMIENTO ONCOLÓGICO:

\section{OTROS TRATAMIENTOS:}

ALBÚMINA antes del tratamiento oncológico:
PREALBÚMINA tras el tratamiento oncológico: g/dl $\mathrm{mg} / \mathrm{dl}$

\section{EXPLORACIÓN FÍSICA:}

Pérdida del tejido adiposo:

$$
\begin{aligned}
& \square \text { Sí. Grado } \\
& \square \text { NO }
\end{aligned}
$$

Pérdida del masa muscular:

$$
\begin{aligned}
& \square \text { Sí. Grado } \\
& \square \text { NO }
\end{aligned}
$$

Edemas y/o ascitis:

$$
\begin{aligned}
& \square \text { sí. Grado } \\
& \square \text { NO }
\end{aligned}
$$

Úlceras por presión: ___ Sí ___ NO

Fiebre: _ sí _ _ NO

Fig. 1. Valoración global subjetiva generada por el paciente. (Versión del Grupo Español de Nutrición y Cáncer ${ }^{5}$ ) 
Por último, la proteína transportadora del retinol (RBP) posee una vida media muy corta (12 horas). Sus niveles disminuyen de forma paralela a los niveles de vitamina A y de zinc, así como en el hipertiroidismo y tras intervenciones quirúrgicas. Su corta vida media y su baja especificidad hacen que se emplee con menos frecuencia que las anteriores. En la Tabla I se exponen sus vidas medias y niveles plasmáticos.

Los métodos antropométricos o tradicionales requieren cierto entrenamiento para la medición de los parámetros y experiencia suficiente para la interpretación de los resultados. Habitualmente son realizados por personal especializado de una Unidad o Servicio de Nutrición.

\section{Métodos rápidos de valoración del estado nutricional}

Actualmente se emplean métodos de cribado o diagnóstico rápido de malnutrición, basados en la inclusión de preguntas sobre hábitos alimentarios, además de medidas antropométricas comunes y determinaciones bioquímicas básicas. Estos métodos son más sencillos y rápidos de realizar, de resultados reproducibles y con poca variación interobservador, de tal forma que personal suficientemente entrenado de un servicio de oncología puede llevarlos a cabo. Para que sean válidos como métodos de cribado o screening deben ser altamente sensibles, es decir, que diagnostiquen a todos los paciente desnutridos o en riesgo de estarlo.

La escala de Valoración Global Subjetiva, desarrollada en los años 80 en el Hospital General de Toronto ha mostrado una sensibilidad y especificidad superiores incluso a parámetros tradicionales como los niveles de albúmina y transferrina ${ }^{8}$. Más tarde fue modificada por Ottery y adaptada a los pacientes con cáncer ${ }^{9}$. En la Valoración Global Subjetiva Generada por el Paciente (VGS-GP) es el propio paciente el que cumplimenta la primera parte del cuestionario, que está referida a la historia clínica, ingesta dietética, síntomas nutricionales y capacidad funcional, mientras que el médico rellena la segunda parte que hace referencia a los signos físicos (Fig. 1). De esta forma se consigue, además, disminuir el tiempo necesario para su realización. Ésta es una diferencia básica con la VGS tradicional, donde es el médico el encargado de completar todo el cuestionario. Esta escala puede realizarse en todos los ámbitos médicos, tanto en pacientes hospitalizados como ambulantes, en consultas externas o en el propio domicilio del paciente. La utilización sistemática de este método permite identificar a los pacientes con malnutrición y valorar los resultados de las intervenciones nutricionales aplicadas en ellos ${ }^{10}$.

La pérdida de peso es uno de los parámetros más relevantes de la escala y se obtiene restando el peso actual del peso habitual o tres meses antes. El médico debe incluir datos sobre el diagnóstico oncológico principal, enfermedades concomitantes y tratamiento oncológico planeado con el fin de valorar el riesgo nutricional del mismo (Tabla II). También se deben enumerar otros tratamientos asociados, como los corticoides o anabolizantes, valores de albúmina o prealbúmina, según el caso, y datos de la exploración física centrada en la pérdida de tejido adiposo y masa muscular y la presencia de edemas o ascitis.
TABLA II

\section{Clasificación de los tratamientos oncológicos según su riesgo nutricional}

Terapia de bajo riesgo nutricional

Radioterapia de mama, SNC, ósea, muscular

Monoquimioterapia a base de MTX, 5-FU bolo, capecitabina

Cirugía menor paliativa

Terapia de riesgo nutricional moderado

Radioterapia pélvica y torácica

Poliquimioterapia, cisplatino, antraciclinas, taxanos, 5-FU i.c.

Ciclofosfamida, Irinotecán

Cirugía colorectal

Terapia de alto riesgo nutricional

Radioterapia de cabeza y cuello, esófago, abdomen superior

Radioquimioterapia concomitante de CyC, pulmón, pelvis

Irradiación corporal total, transplante de médula ósea

Cirugía oncológica de CyC y aparato digestivo

SNC: Sistema Nervioso Central; MTX: Metotrexato; 5-FU: 5-fluorouracilo; CyC: Cabeza y cuello.

Para valorar la pérdida de tejido adiposo se pueden explorar la zona inferior del ojo, la zona anterior de la parrilla costal inferior o la parte posterior del brazo, cogiendo un pellizco de piel a este nivel. Una pérdida importante de tejido adiposo subcutáneo indica, generalmente, un déficit energético severo. Para la valoración de la masa muscular se debe apreciar el volumen y el tono en el músculo temporal, en los hombros, en los muslos o en la zona gemelar. Por último se debe prestar atención a la existencia de edemas o ascitis que se pueden presentar en la malnutrición, aunque habría que descartar fallo hepático o carcinomatosis peritoneal.

\section{Evaluación de los resultados de la VGS-GP}

Los datos obtenidos de la escala, tanto generados por el paciente como por el médico evaluador, se transfieren a la tabla de valoración global (Fig. 2). Al final del proceso tendremos 12 evaluaciones parciales y la valoración global será la que predomine de las tres columnas: buen estado nutricional, malnutrición moderada, de riesgo de malnutrición o malnutrición grave. Si, por ejemplo tenemos 8 evaluaciones parciales en la columna A, 2 en la B y 2 en la C, la valoración global será $\mathrm{A}$ : buen estado nutricional. $\mathrm{Si}$, por el contrario tenemos 5 en la columna $B$ y 7 en la $C$ diremos que el paciente presenta una malnutrición grave. Hay dos parámetros, sin embargo, que sitúan al paciente por sí mismos en la columna $C$ de malnutrición grave: la pérdida de peso superior al $10 \%$ y la albúmina o prealbúmina por debajo de los niveles críticos.

Se ha demostrado que este método tiene poca variabilidad interobservador por lo cual sus resultados son fácilmente reproducibles, siempre que los evaluadores tengan cierta ex- 


\section{VALORACIÓN GLOBAL}

Teniendo en cuenta el formulario, señale lo que corresponda a cada dato clínico para realizar la evaluación final:

\section{DATO CLÍNICO}

\section{Pérdida de peso}

Alimentación

Impedimentos para ingesta

Deterioro de actividad

Edad

Úlceras por presión

Fiebre / corticoides

Tto antineoplásico

Pérdida adiposa

Pérdida muscular

Edemas / ascitis

Albúmina (previa al tratamiento)

Prealbúmina (tras el tratamiento)
A

$<5 \%$

Normal

NO

NO

$£ 65$

NO

NO

bajo riesgo

NO

NO

$\mathrm{NO}$

$>3^{\prime} 5$

$>18$
B

\section{5-10\%}

deterioro leve-moderado

leves-moderados

leve-moderado

$>65$

NO

leve / moderada

medio riesgo

leve / moderada

leve / moderada

leve / moderados

3'0-3'5

15-18
C

$>10 \%$

deterioro grave

graves

grave

$>65$

sí

elevada

alto riesgo

elevada

elevada

importantes

$<3^{\prime} 0$

$<15$

VALORACIÓN GLOBAL:

$A$ : buen estado nutricional

B: malnutrición moderada o riesgo de malnutrición

C: malnutrición grave

Fig. 2. Evaluación de los resultados de la VGS-GP5.

periencia. Por otra parte también ha demostrado ser un método más sensible que la antropometría ${ }^{10}$. Estas propiedades la convierten en un excelente método de cribado de malnutrición.

\section{Impacto de una correcta intervención nutricional en oncología}

La identificación del estado nutricional basal de un paciente con cáncer puede tener impacto en el éxito del tratamiento antineoplásico y en su pronóstico general. Una vez realizada la valoración nutricional global del paciente debemos aplicar el soporte nutricional apropiado en cada caso. Para ello seguiremos los protocolos de actuación o algoritmo de intervención nutricional adaptado a cada servicio o centro sanitario. Para los pacientes con buen estado nutricional al diagnóstico y que vayan a recibir tratamiento oncológico de bajo o moderado riesgo sería suficiente, en principio, con una correcta información sobre los posibles efectos secundarios del tratamiento y una serie de recomendaciones nutricionales. El consejo dietético suele ser muy apreciado por pacientes y familiares y para poder realizarlo de forma sistemática, la ayuda de enfermeras, dietistas y personal asociado es inestimable. La información escrita en forma de guías o folletos puede facilitar esta tarea.

Para el segundo grupo de pacientes, aquellos bien nutridos pero que van a ser sometidos a terapia oncológica de alto riesgo, sería beneficioso forzar la ingesta calórica en el intervalo previo al tratamiento. La prescripción de suplementos orales puede ayudar a mantener el peso durante el tratamiento oncológico dentro de unos límites aceptables. El soporte nutricional tiene un impacto positivo en los pacientes ambulatorios en tratamiento radioterápico y concretamente en los afectos de tumores de cabeza y cuello la intervención nutricional precoz es eficaz en la prevención de complicaciones y en la mejoría de la calidad de vida de los pacientes ${ }^{11}$. Algunos autores preconizan incluso instaurar alimentación artificial profiláctica mediante sonda nasogástrica o gastrostomía endoscópica percutánea ${ }^{12,13}$. Estas medidas van encaminadas a asegurar el cumplimiento del protocolo terapéutico específico, por ejemplo radioquimioterapia en pacientes con cáncer de cabeza y cuello, minimizando el riesgo de complicaciones y manteniendo la calidad de vida del paciente.

Por último para los pacientes malnutridos el soporte nutricional activo puede permitir en algunos casos iniciar el tratamiento oncológico, que de otra forma, habría que desestimar. En estos casos la nutrición enteral es de elección en los pacientes malnutridos que son incapaces de mantener una ingesta oral adecuada. La elección de sonda nasogástrica, gastrostomía o yeyunostomía variará en función de la situación particular del paciente, de su enfermedad y de la duración estimada del soporte nutricional. Las indicaciones de nutrición parenteral quedarían restringidas a situaciones postoperatorias y subgrupos especiales de enfermos con alteraciones intestinales graves que invaliden la vía entera |14, 15.

La consecuencia esperable de mejorar el estado nutricional y la tolerancia al tratamiento oncológico específico mediante soporte nutricional sería el aumento de supervivencia en estos pacientes. Aunque sí se ha demostrado que los pacientes con mayor porcentaje de pérdida de peso presentan una supervivencia significativamente menor ${ }^{2}$, lo anterior es más difícil de demostrar. Quizás la pérdida de peso sólo sea un marcador de enfermedad más agresiva, al igual que lo es la presencia de anemia o de síntomas constitucionales.

Sin embargo, la intervención nutricional desde el diagnós- 


\section{Cerezo}

tico puede ayudar a mantener el peso, aumentar la respuesta al tratamiento, disminuir la incidencia de complicaciones y mejorar la calidad de vida tanto del paciente como de sus familiares, que a menudo se sienten preocupados por el peso y los hábitos alimenticios del paciente ${ }^{16}$. Como es fácilmente deducible no todos los pacientes que reciben radioterapia o quimioterapia necesitan soporte nutricional. Lo importante es, por tanto, seleccionar bien a los pacientes que puedan beneficiarse del soporte nutricional ${ }^{17}$.

\section{Conclusiones}

Parece evidente que el soporte nutricional puede mejorar la tolerancia a los tratamientos oncológicos específicos y por tanto mejorar la calidad de vida en el paciente oncológico.

Es precisa una intervención nutricional precoz, mediante un abordaje multidisciplinario que implique al oncólogo clínico, al especialista en nutrición, al paciente y a su familia.

La escala de Valoración Subjetiva Global Generada por el Paciente es un método válido de evaluación inicial. Este método es sensible, específico y tiene poca variabilidad entre observadores, si han sido adecuadamente entrenados.

La experiencia acumulada en los últimos años nos permite concluir que existe una relación significativa entre pérdida de peso y menor supervivencia. Sería conveniente investigar si al revertir la pérdida de peso mediante soporte nutricional apropiado aumentarán las tasas de supervivencia en los pacientes oncológicos.

\section{Bibliografía}

1. Ottery FD. Rethinking nutritional support of the cancer patient: The new field of nutritional oncology. Semin Oncol 1994; $21: 770-778$.

2. De Wys WD, Begg C, Lavin PT et al. Prognostic effect of weight loss prior to chemotherapy in cancer patients. Am J Med 1980; 69:491-497.

3. Langer CJ, Hoffman JP, Ottery FD. Clinical significance of weight loss in cancer patients: rationale for the use of anabolic agents in the treatment of cancer-related cachexia. Nutrition 2001; 17 (Suppl1):S1-20.

4. Hill GL. Body composition research: implications for the practice of clinical nutrition. JPEN 1992; 16:197-218.

5. Luengo LM. Valoración del estado nutricional del paciente oncológico. Rev Oncol 2004: 6 (Supl 1): 11-18.

6. Kalzarentzos F, Spiliotis J, Velimenzis G. Comparison of forearm muscle dynamometry with nutritional prognostic index, as a preoperative indicator in cancer patients. JPEN 1989; 13:34-36.

7. Inoue $Y, N e z u R$, Matsuda $H$, et al. Rapid turnover proteins as a prognostic indicator in cancer patients. Surg Today 1995; 25:498-506.

8. Baker JP, Destky AS, Wesson DE, et al. Nutritional assessment: a comparison of clinical judgement and objective measures. $\mathrm{N}$ Engl J Med 1982; 306:969-972.

9. Ottery FD. Oncology patient-generated SGA of nutritional status. Nutr Oncol 1994; 1(2):9.

10. Gómez-Candela C, Luengo LM, Cos AL, et al. Valoración subjetiva global en el paciente neoplásico desarrollada por Servicios de Nutrición vs Oncología. ¿̇Es siempre útil para evaluar el estado nutricional? Nutr Hosp 2003; 18:353-357.

11. Piquet MA, Ozsahin M, Larpin I, et al. Early nutritional intervention in oropharyngeal cancer patients undergoing radiotherapy. Support Care Cancr 2002; 10:502-504.

12. Mekhail TM, Adelstein DJ, Rybicki LA, et al. Enteral nutrition during the treatment of head and neck carcinoma: is a percutaneous endoscopic gastrostomy tube preferable to a nasogastric tube? Cancer 2001; 91:1785-90.

13. Piquet MA, Ozsahin M, Larpin I, et al. Early nutritional intervention in oropharyngeal cancer patients undergoing radiotherapy. Support Care Cancer 2002; 10:502-504.

14. McGeer AJ, Detsky AS, O'Rourke K. Parenteral nutrition in cancer patients undergoing chemotherapy: a metaanalysis. Nutrition 1990; 6:233-40

15. Sikora SS, Ribeiro $U$, Kane $J M$, et al. Role of nutrition support during induction chemoradiation therapy in esophageal cancer. J Parenter Enteral Nutr 1998; 22:18-21.

16. McCowen KC, Bristriam BR. Immunonutrition: problematic or problem solving? Am J Clin Nutr 2003; 77:764-70.

17. García P. Estrategia nutricional en el paciente oncológico. Rev Oncol 2004: 6 (Supl 1):40-45. 